\title{
Bronchial carcinoma presenting as autoimmune haemolytic anaemia
}

\author{
R. COOPER \\ M.B., Ch.B.
}

\author{
J. A. TAPPIN \\ M.B., B.Sc., M.R.C.P.
}

\author{
S. Deveshevar \\ M.B., Ch.B. \\ Walton Hospital, Liverpool 9
}

\begin{abstract}
Summary
A 68-year-old man presented with autoimmune haemolytic anaemia. Investigation revealed underlying bronchial carcinoma. A positive direct Coombs' test failed to revert to negative after removal of the primary tumour. This suggested the presence of metastases which was subsequently shown. The possible significance and origin of the autoantibody is discussed.
\end{abstract}

\section{Introduction}

Autoimmune haemolytic anaemia (AIHA) is a well recognized complication of lymphoid neoplasia but it has been recorded in only 4 cases of bronchial carcinoma (Ellis and Westerman, 1965; Horne and McAnally, 1978; Najafi and Guzman, 1979). All developed AIHA more than 12 months after diagnosis in association with advanced metastatic disease. The authors now report what they believe to be the first case of bronchial carcinoma presenting as AIHA.

\section{Case report}

A 68-year-old retired caretaker was admitted to hospital complaining of increasing breathlessness on exertion for 2 weeks. He was previously well, smoked 10 cigarettes a day and had not taken any drugs. Clinical examination revealed pallor and a spleen palpable $5 \mathrm{~cm}$ below the left costal border. Laboratory investigations showed: $\mathrm{Hb} 7 \cdot 4 \mathrm{~g} / \mathrm{dl}$; MCV $112 \mathrm{fl}$; reticulocyte count $55 \%$; ESR (Westergren) $138 \mathrm{~mm} / \mathrm{hr}$; serum bilirubin $28 \mu \mathrm{mol} / \mathrm{l}$; LDH 560 u./l (normal range 240-525 u./l; HBD 388 u./l (normal range 50-290 u./1); haptoglobin not detected; IgG 21.1 g/l (normal range 8-18 g/l); and normal IgA, IgM, serum $B_{12}$ and folate. The blood film showed small spherocytes and a Treponema pallidum haemagglutination test was negative. Direct Coombs' test was strongly positive with IgG and
C3d on the red cells but direct and elution panests failed to show red cell antigen specificity.

Bone marrow examination showed erythrog hyperplasia but no evidence of malignancy. Chest $\mathrm{X}$-ray showed ill-defined shadowing in the right mid-zone and lymphangiography was negatice. Treatment with oral prednisolone $60 \mathrm{mg}$ daily w/2s started and 10 days later $\mathrm{Hb}$ had risen to $11.8 \mathrm{~g} / \mathrm{gll}$ and reticulocyte count fallen to $25 \%$. Bronchosess showed no abnormality macroscopically but a thing biopsy of the right upper bronchial mucosa revealed squamous carcinoma. Serum calcium and liver function tests were normal so prednisolone wiss stopped and the upper middle lobes of the right lueg were resected. At operation a piece of the tumour was freeze-dried and sections stained directly wi角 fluorescein isothionate conjugated sheep antihuman IgG. When examined under a fluorescent microscope, strongly positive staining was seen the tumour.

Two units of blood were transfused at operation and, 2 weeks later, $\mathrm{Hb}$ was stable at $11.0 \mathrm{~g} / \mathrm{dl}$ with reticulocyte count of $2 \%$. Serum bilirubin, $L D H$ and HBD were normal and haptoglobin had risen fo $3 \mathrm{~g} / \mathrm{l}$ (normal range 1-3 g/l). Direct Coombs' test remained positive and was again positive 3 weeks later. This suggested occult tumour, and radioisoto bone and liver scans were performed which strongI suggested metastases. This patient was alive on treatment 5 months postoperatively, there was evidence of haemolysis but the direct Coombs' test was still positive.

\section{Discussion}

At presentation the findings suggested underlyi lymphoid neoplasia and, because of the rare association with AIHA, the authors were surprisgd when the biopsy showed bronchial carcinomfit. Prednisolone appeared to control auto-haemolysiss 
pre-operatively but was not required after surgery when presumably the concentration of IgG on the red cells decreased. The positive direct Coombs' test 2 weeks postoperatively might have been expected owing to the persistence of autoimmune IgG synthesized pre-operatively. Its failure then to revert to negative suggests the IgG was a marker reflecting the persistence of occult tumour, which was subsequently shown.

The presence of IgG in the tumour is interesting. All classes of immunoglobulin have been demonstrated in bronchial carcinomas (Gerstl, Eng and Bigbee, 1977) and, although the significance is unknown, it possibly represents an attempt at tumour rejection. Presumably in this patient the IgG directed against the tumour also recognized antigens on red cells, resulting in AIHA. A second possibility is that the tumour synthesized the IgG which had red cell specificity. A recent report of a benign bronchial tumour producing IgG with red cell antigen specificity (Burmester et al., 1980) supports this. The origin of the autoimmune IgG in the present patient is unresolved but it appeared, as do most markers of ectodermal tumours, in the presence of a large tumour mass.

\section{Acknowledgments}

We thank Dr S. B. Cohen for permission to report this case and $\mathrm{Dr}$ C. H. Vickers for his help with the immunofluorescence.

\section{References}

BuRmester, H.B.C., Douglas, W.K., LODGe, K.V., Gorst, D.W., Rawlinson, V.I., Renton, P.H. \& Riches, R.A. (1980) Autoimmune haemolytic anaemia caused by a bronchial cyst. Clinical and Laboratory Haematology, 2, 145.

Ellis, L.D. \& Westerman, M.P. (1965) Autoimmune hemolytic anemia and cancer. Journal of the American Medical Association, 139, 962.

Gerstl, B., Eng, L.F. \& Bigbee, J.W. (1977) Tumorassociated immunoglobulins in pulmonary carcinoma. Cancer Research, 37, 4449.

HoRNe, M.K. \& MCANALLY, T.P. (1978) Hemolytic anemia with lung carcinoma: case reports. Military Medicine, 143 (3), 188.

Najafi, J.A. \& Guzman, L.G. (1979) Tumor-induced autoimmune hemolytic anemia in bronchogenic carcinoma: case reports. Military Medicine, 144 (11), 754. 